\title{
Pedestrian Detection Approach for Driver Assisted System using Haar based Cascade Classifiers
}

\author{
M. Ameen Chhajro ${ }^{1}$, Kamlesh Kumar ${ }^{2}$, M. Malook \\ Rind $^{3}$, Aftab Ahmed Shaikh ${ }^{4}$, Haque Nawaz ${ }^{5}$ \\ Department of Computer Science \\ Sindh Madressatul Islam University, Karachi, Sindh, Pakistan
}

\author{
Rafaqat Hussain Arain ${ }^{6}$ \\ Department of Computer Science \\ Shah Abdul Latif University, Khairpur, Sindh, Pakistan
}

\begin{abstract}
Object detection and tracking with the aid of computer vision is a most challenging task in the context of Driver Assistant System (DAS) for vehicles. This paper presents pedestrians detection techique using Haar-Like Features. The main aim of this research is to develop a detection system for vehicle drivers that will intimate them in advance for pedestrian's movement when they are crossing the zebra region or passing nearby to it along the road. For this purpose, dataset of 1000 images have been taken via CCTV camera which was mounted for road monitoring. A Haar based cascade classifiers have been implemented over images. And system is trained for positive (with people) and negative (without people) image samples, respectively. After testing, the obtained results show that it attained $90 \%$ accuracy while pedestrian detection. The proposed work provides significant contribution in order to reduce the road accidents as well as ensure the safety measurement for road management.
\end{abstract}

Keywords-Pedestrian; Haar based classifier; positive and negative samples; computer vision; object detection

\section{INTRODUCTION}

During past few years, field of computer vision for the object detection and tracking has been a hotspot area for researchers. This has become due to growing interest in visual data; as to acquire hidden patterns that could enable machines for automated decision making tasks. And it might be explored through numerous applications which proves the potential benefits of this research domain, e.g. Road Sign Recognition, Passive surveillance, License Plate Recognition, Face Tracking, Pedestrian Tracking and beside other related area [1]-[6]. Detecting pedestrian remains an ineluctable task for driver assistance system. However, pedestrian movement recognition is also a crucial research problem that cannot be overlooked. Therefore, it is matter of more attention to detect pedestrian as to avoid any adverse accident and to control the speed of vehicle. In this paper, a vision based driving assistant system has been proposed which aims to detect people which are near to or crossing the zebra region. This work provides significant contribution over people safety.

The rest of the paper is arranged as follows. Section II gives literature review related to pedestrian detection. Section III explains training procedure of cascading classifier for tracking objects in an image. Section IV provides simulation results and discussion over zebra crossing people detection. Finally, Section V gives the conclusion and future work.

\section{LITERATURE REVIEW}

Over last few years, various works has been reported in pedestrian detection for driver assistance system. In this regard, J. Hariyono and K. Hyun Jo suggested [7] a model for detection of pedestrian crossing the road. In which pedestrian pose and lateral speed are recognized using spatial body mass ratio. Whereas, motion trajectory and spatial layout are obtained through centroid of human region and distance of the pedestrian along the road lane boundary. Similarly, B. Riveiro et al. [8] proposed mobile LIDAR data technique for automatic detection of zebra crossing. It uses several successive processes, begins with segmentation of road for curvature analysis in each laser cycle. Then, implements rasterization and Standard Hough Transform techniques, in order to detect zebra crossing. This work is beneficial for road managers that need Geographic Information Systems. Object detection method for advanced driver assistance systems (ADAS) was presented by M. Kang and Y.C Lim [9]. In this research, authors employ fully convolutional network (FCN) in order to detect objects in road environment. In a similar context, B. Wang et al. [10] provided an approach for multiple object tracking and detection for road obstacles. Their system works well when this was tested on different traffic video sequences over public database. Sanchez et al. [11] used Convolutional Neural Network for pedestrian movement and direction recognition, in which histograms of gradients (HOG) and SVM have been implemented for pedestrian detection. Another paper C. Caramuta et al. [12] provided comprehensive survey over pedestrian dynamics detection techniques, simulation and mathematical models. Similarly, Hui Zhou and Wan Hang [13] demonstrated Lane Detection and Tracking for Driver Assistance Systems.

\section{CASCADING CLASSIFIERS}

The cascade classifiers are special case of ensemble learning, where several classifiers are combined together [14]. These are mostly used for object detection from images and it was firstly proposed by Viola and Jones in 2001 for face detection. The cascade classifiers are trained for positive and negative images for specific object detection. However, training phase of classifier requires tradeoff between feature selection and its computation. For example, classifier with 
more features produces higher detection rates as compared to false positive results. But on the other side, it increases its computation time. Therefore, optimization of classifier depends upon selection of framework for training. For this purpose, one must consider following parameters. These are 1) number of classifier stages, 2) the number of features in each stage, and 3) threshold in each stage. In fact, an effective classifier is one that could work well in real time object detection, and such kind of classifiers are very difficult to design. However, cascade classifiers under goes subsequent training stages for target selection. And during each stage in the cascade, it reduces the false positive rate and increases the detection rate. Features are added to a classifier in each stage, until we get targeted object along with reduction of false positives rates. And, accuracy of the classifier is determined by testing it on a ground truth data.

\section{Proposed Methodology}

In this paper, Haar Based Cascade Classifier has been implemented for object detection. This classifier has been used successfully for detection and tracking of objects in an image [15]. In order to train the classifier, an image sample is built for positive and negative images respectively for targeted and unwanted objects. The main aim of using classifier is to generate most optimized targeted values for detecting and tracking the object through varying the size of window for Haar feature selection as highlighted in Fig. 1. It filters the features of positive images and then creates specific target values through separation of black and white areas in the image features. And these are placed in an image where we want to perform object detection.

In an image there are different objects, but we are interested in finding the specific object from it. For this purpose, a training is carried out over dataset for positive and negative images for object location and detection. Testing of the classifier has been achieved for positive (with people) and negative (without people) images as shown in Fig. 2 and 3 respectively. These images are obtained via video sequence using a fixed CCTV Camera. And it is mounted on the road for monitoring the traffic. We have made dataset of 1000 images, each having 500 images for positive and negative samples, respectively. Thus classifier executes as aforementioned manner, however, speed of locating the objects in the image highly depends upon classifier training.
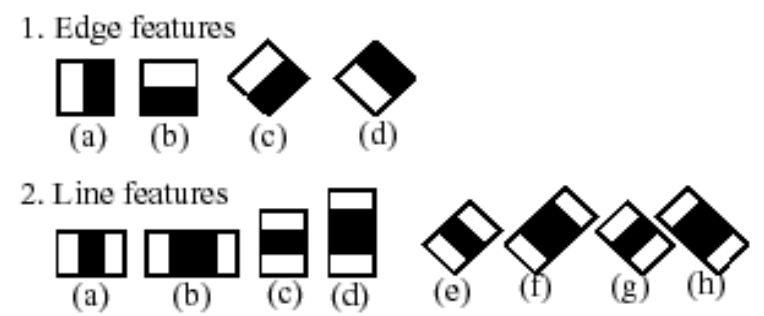

3. Center-surround features

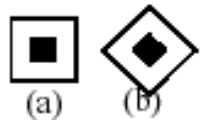

Fig. 1. Types of Haar features.

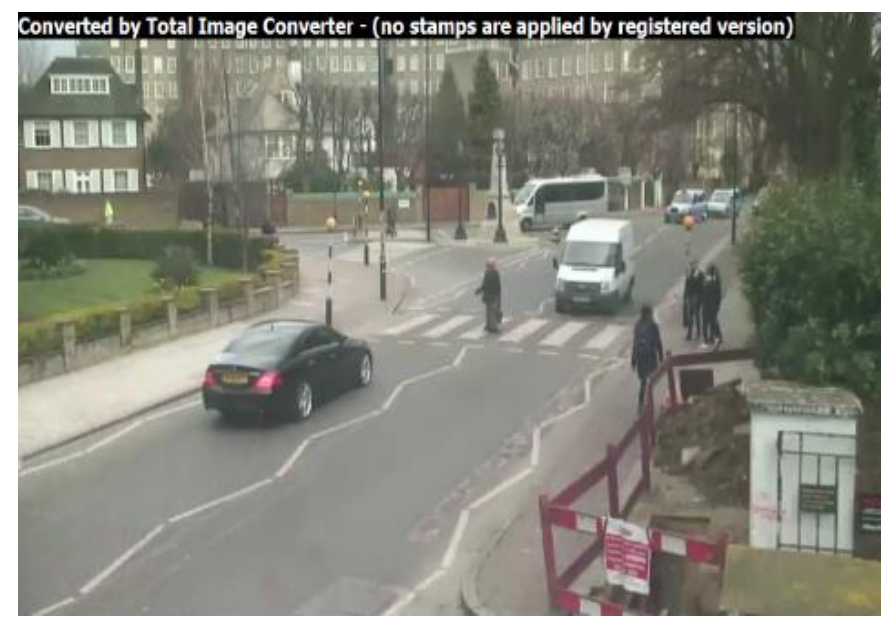

Fig. 2. Positive sample image.

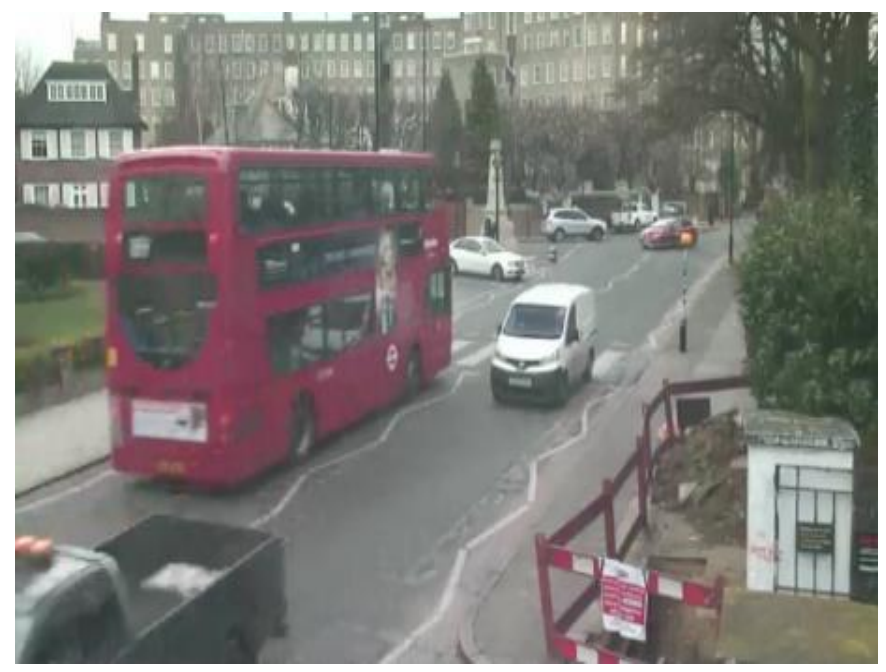

Fig. 3. Negative sample image.

\section{Simulation Results AND Discussions}

In this section, simulation results are shown for proposed system and discussion is made over it. In this regard, testing is performed for some positive and negative images for people detection. However, research aim is to detect people which are near or over the zebra crossing region. For this purpose, we have taken 300 images for training, and then performed testing over 100 images. The performance of proposed system has been evaluated using correct and mis-detection metrics.

Table I depicts that system recognize 90 images with people out of 100 and leaving only 10 mis-identified images. It can be observed from Fig. 4 and 5, respectively, there are different objects namely: people, vehicles, poles, buildings, barriers and trees. But proposed system is trained to detect people when they are crossing the zebra or approaching near to it. However, in some cases it yields mis-detection, when both the people and beside other surrounding object are targeted as shown in Fig. 6. The graphical results in Fig. 7 shows that proposed system provides higher recognition rates as compared to mis-detection as it achieves accuracy of $90 \%$ when tested over dataset. 
TABLE I. RESUlts OUTCOMES OF PROPOSED MOdEL

\begin{tabular}{|l|l|l|l|l|l|}
\hline $\begin{array}{l}\text { No. of } \\
\text { Images } \\
\text { in } \\
\text { Dataset }\end{array}$ & $\begin{array}{l}\text { No. of } \\
\text { Images for } \\
\text { Training }\end{array}$ & $\begin{array}{l}\text { No. of } \\
\text { Images } \\
\text { for } \\
\text { Testing }\end{array}$ & $\begin{array}{l}\text { Correctly } \\
\text { Detected }\end{array}$ & $\begin{array}{l}\text { Mis- } \\
\text { Detected }\end{array}$ & Rate \\
$(\%)$
\end{tabular}

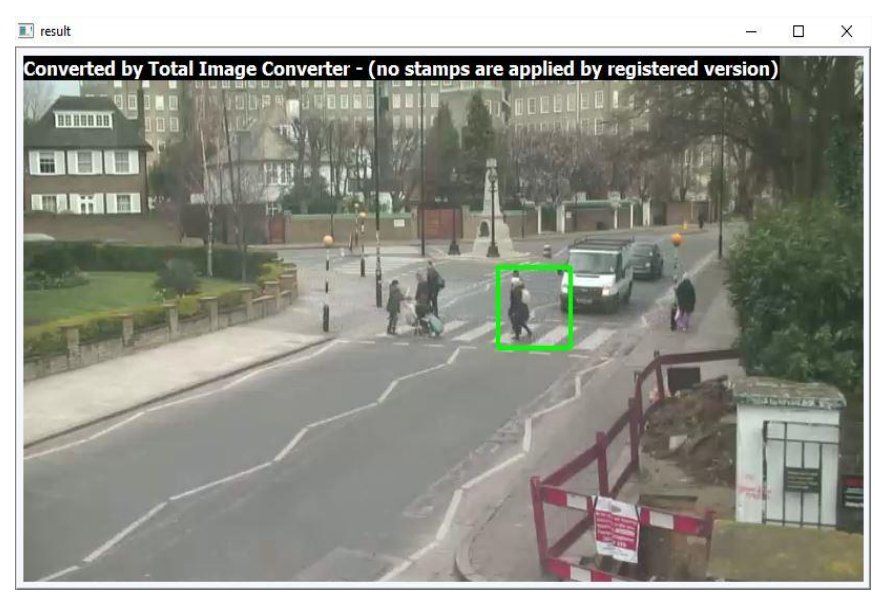

Fig. 4. Pedestrian detection along zebra crossing.

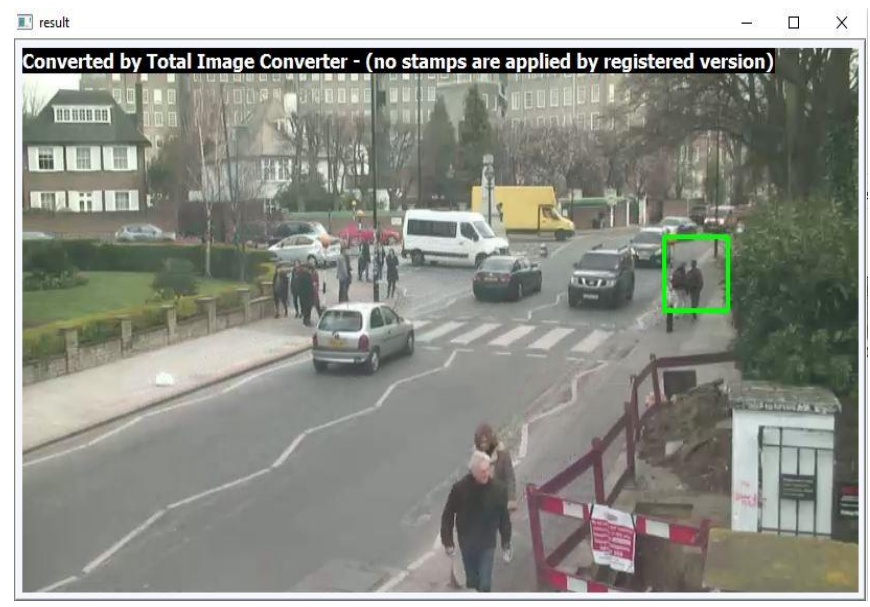

Fig. 5. Pedestrian detection near zebra crossing.

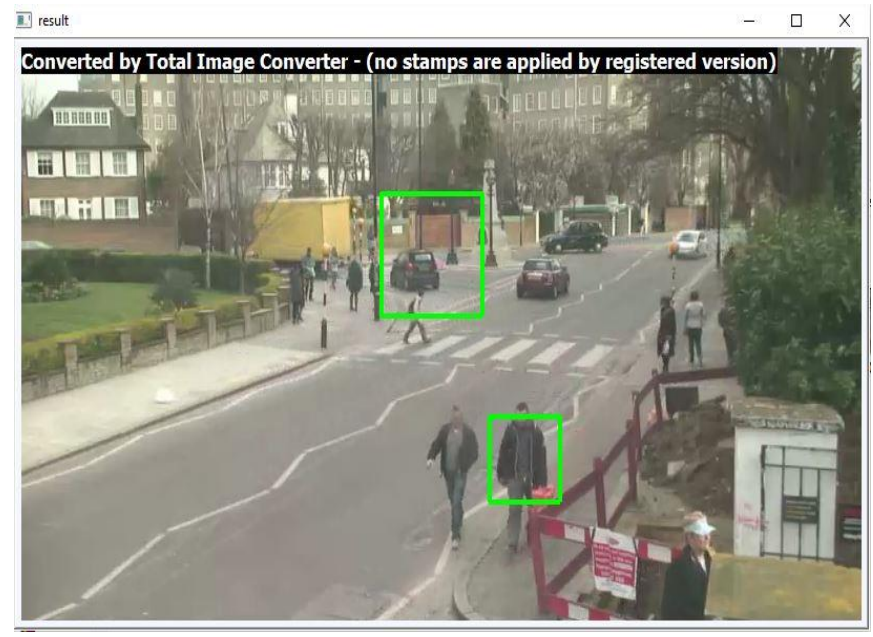

Fig. 6. Pedestrian mis-detection.

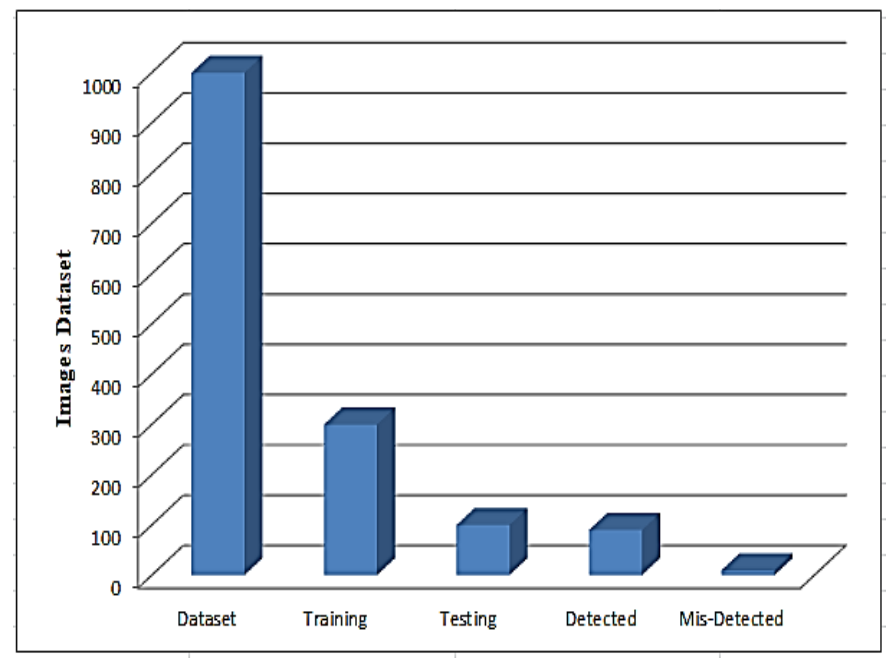

Fig. 7. Graphical results representation.

\section{CONCLUSION AND FUTURE WORK}

Accurate pedestrian detection and tracking remains a big dilemma in computer vision for driver assisted system. In this paper, people detection near to or crossing the zebra region was proposed for vehicle drivers. A Haar based cascade classifiers were used for feature detection for positive and negative samples respectively. The system was trained over 1000 images obtained via CCTV camera. After testing of 100 images, results showed that out of them 90 images were correctly identified leaving only 10 mis-identified images. However, overall system attained accuracy of $90 \%$. In future, we shall improve the performance of this algorithm using deep learning technique for classification in order to improve its accuracy.

\section{REFERENCES}

[1] Chen, Y., Zhao, D., Lv, L. and Zhang, Q., 2017. Multi-task learning for dangerous object detection in autonomous driving. Information Sciences.

[2] Dominguez-Sanchez, A., Cazorla, M. and Orts-Escolano, S., 2017. Pedestrian Movement Direction Recognition Using Convolutional Neural Networks. IEEE Transactions on Intelligent Transportation Systems, 18(12), pp.3540-3548.

[3] Zhong, Z., Lei, M., Cao, D., Fan, J. and Li, S., 2017. Class-specific object proposals re-ranking for object detection in automatic driving. Neurocomputing, 242, pp.187-194

[4] Talib, H., Ismail, K. and Kassim, A., 2016. A robust approach for road users classification using the motion cues. Transportation Research Part C: Emerging Technologies, 73, pp.77-90.

[5] Wang, B., Florez, S.A.R. and Frémont, V., 2014, December. Multiple obstacle detection and tracking using stereo vision: application and analysis. In Control Automation Robotics \& Vision (ICARCV), 2014 13th International Conference on (pp. 1074-1079). IEEE.

[6] Wang, S., Pan, H., Zhang, C. and Tian, Y., 2014. RGB-D image-based detection of stairs, pedestrian crosswalks and traffic signs. Journal of Visual Communication and Image Representation, 25(2), pp.263-272.

[7] J. Hariyono, K. H, Jo. Detection of Pedestrian Crossing Road A Study on Pedestrian Pose Recognition [J]. Neurocomputing, 2016, 234, 2017, 144-153

[8] B. Riveiroa, H. G. Jorge, J. M. Sánchez, L. D. Vilariño, P. Arias. Automatic detection of zebra crossings from mobile LiDAR data[J]. Optics \& Laser Technology, 2015, 70, 63-70

[9] M. S. Kang, Y. C. Lim. High Performance and fast objection in road environment $[\mathrm{C}]$. International Conference on Image Processing Theory, Canada, 2017, 1-6 
[10] Wang, B., Florez, S.A.R. and Frémont, V., 2014, December. Multiple obstacle detection and tracking using stereo vision: application and analysis. In Control Automation Robotics \& Vision (ICARCV), 2014 13th International Conference on (pp. 1074-1079). IEEE.

[11] Dominguez-Sanchez, A., Cazorla, M. and Orts-Escolano, S., 2017. Pedestrian Movement Direction Recognition Using Convolutional Neural Networks. IEEE Transactions on Intelligent Transportation Systems, 18(12), pp.3540-3548.

[12] Caramuta, C., Collodel, G., Giacomini, C., Gruden, C., Longo, G. and Piccolotto, P., 2017. Survey of detection techniques, mathematical models and simulation software in pedestrian dynamics. Transportation research procedia, 25 , pp.551-567.
[13] Zhou, H. and Wang, H., 2017, November. Vision-based lane detection and tracking for driver assistance systems: A survey. In Cybernetics and Intelligent Systems (CIS) and IEEE Conference on Robotics, Automation and Mechatronics (RAM), 2017 IEEE International Conference on (pp. 660-665). IEEE.

[14] Kuo, Y.C., Yang, Z.Y. and Yen, C.H., 2012. Fast pedestrian detection system with a two layer cascade of classifiers. Computers \& Mathematics with Applications, 64(5), pp.1311-1323.

[15] Mohamed, A. Issam, A. Mohamed, B. and Abdellatif, B., 2015. Realtime detection of vehicles using the haar-like features and artificial neuron networks. Procedia Computer Science, 73, pp.24-31. 